\title{
The Extragalactic Background Light: The Emission from All Stars and Black Holes
}

\author{
Sara Buson*t \\ Università di Padova, Dip. di Fisica e Astronomia “G. Galilei”, I-35131 Padova, Italy \\ INFN, Sezione di Padova, I-35131 Padova, Italy \\ E-mail: sara.busonenasa.gov
}

\section{on behalf of the Fermi-LAT collaboration}

\begin{abstract}
Intergalactic space is filled with the light produced by all the stars and accreting compact objects that populated the observable Universe throughout the whole cosmic history. This relic cosmic background from IR to UV is called the diffuse extragalactic background light (EBL). Knowledge of the EBL and its cosmic evolution represents a key issue in astrophysics since it helps unveiling the nature of star formation and galaxy evolution. Direct measurements of the EBL are difficult due to bright local foregrounds, in particular zodiacal light. As a consequence indirect measurements constitute a mandatory complement to them. A powerful approach for probing these diffuse radiation fields in the UV to far-IR bands is through gamma-gamma absorption of high-energy photons. Pair production $\left(e^{+} e^{-}\right)$from high-energy photons interacting with ultraviolet to infrared EBL photons is effective at attenuating gamma rays with energy above $\sim 10 \mathrm{GeV}$. This process introduces an attenuation in the spectra of gamma-ray sources above a critical energy. The major effect is a redshift- and energy-dependent dimming of the gamma-ray flux of extragalactic sources such as blazars and gamma-ray bursts (GRBs), which can be used to infer limits on the EBL intensity. All recent measurements favor models with a minimal level of EBL, consistent with the estimated contribution from resolved galaxies.
\end{abstract}

Frontier Research in Astrophysics

26 - 31 May, 2014

Mondello (Palermo), Italy

${ }^{*}$ Speaker.

${ }^{\dagger}$ Now at NASA Goddard Space Flight Center/UMBC/CRESST 


\section{Introduction}

The Extragalactic Background Light is the sum of all ultraviolet, optical and infrared radiation emitted over the history of the Universe [1]. It encodes information about the relic emission from the first stars, galaxies and accreting compact objects that subsequently shaped galaxy formation and evolution [2]. Its spectral energy distribution (SED) appears characterized by two main bumps (in the $v-v F_{v}$ representation as seen in Figure 1). The former, located in the optical near- mid-infrared bands, is attributed to direct galaxy starlight, while the second to light reprocessed by dust and re-radiated in the mid- far-infrared bands. Huge efforts have been made to model the EBL, since it provides unique information about the epochs of formation and evolution of galaxies. Nevertheless, absolute measurements still remains challenging, because direct photometry measurements have to deal with difficult foreground subtractions and calibration issues. In general, complex modeling is required to treat the evolution of galaxy populations. Deep surveys (HST/Spitzer) can give reliable lower limits by simply integrating the number counts of resolvable sources available at many wavelengths, but this approach may miss faint source populations.

Importantly, integrating the light from resolved galaxies to the faintest end of the luminosity functions provides a strict lower limit on the EBL abundance. Some studies based on night-sky background have suggested a higher isotropic emission than could be accounted for by the total contribution of galaxies. Although the extragalactic origin of this excess is debatable, especially due to the possible contamination by the intense foreground such as the zodiacal light, this suggestion encouraged the idea of an additional contribution by other sources and in particular by Population III, zero-metallicity stars during the epoch of strong star formation at $z \sim 10$ [3].

Gamma rays offer a unique complement to direct EBL estimates [4, 5]. High-energy (HE) photons above $\mathrm{GeV}$ energies can interact with $\mathrm{EBL}$ radiation through $\mathrm{e}^{ \pm}$pair production following $\mathrm{E}_{\gamma} \times \mathrm{E}_{\mathrm{EBL}} \geq 2\left(\mathrm{~m}_{\mathrm{e}} \mathrm{c}^{2}\right)^{2}$. The more distant the object, the higher the probability that its photons are converted into pairs and, in turn, the greater the attenuation of its gamma-ray spectrum. Distant powerful gamma-ray sources such as active galactic nuclei (AGN), blazars in particular, constitute the ideal means to probe the EBL through this mechanism. There have been a great variety of methodologies intended to exploit the gamma-ray attenuation approach. A comprehensive review on this can be found in [6], while a concise summary about EBL observational strategies is available in [7]. In the following I will present some of the current techniques, based mostly on gamma-ray observations, and discuss also the major problems affecting them.

\section{Constraints from blazar gamma-ray spectra}

One indirect, powerful technique to probe the EBL is based on the fact that gamma rays passing through intergalactic space have a high probability to collide with EBL photons and be converted into electron-positron pairs. The gamma-ray spectrum of a distant source, observed at Earth, is shaped by this interaction. The typical attenuation curve for gamma rays is represented in Figure 2 and implies a particular redshift/energy-dependent attenuation in the spectra of all distant sources. The missing gamma-ray flux can be used as a proxy to derive the EBL abundance, since the interaction implies a simple one-to-one relation between EBL photons and extragalactic gamma rays. 


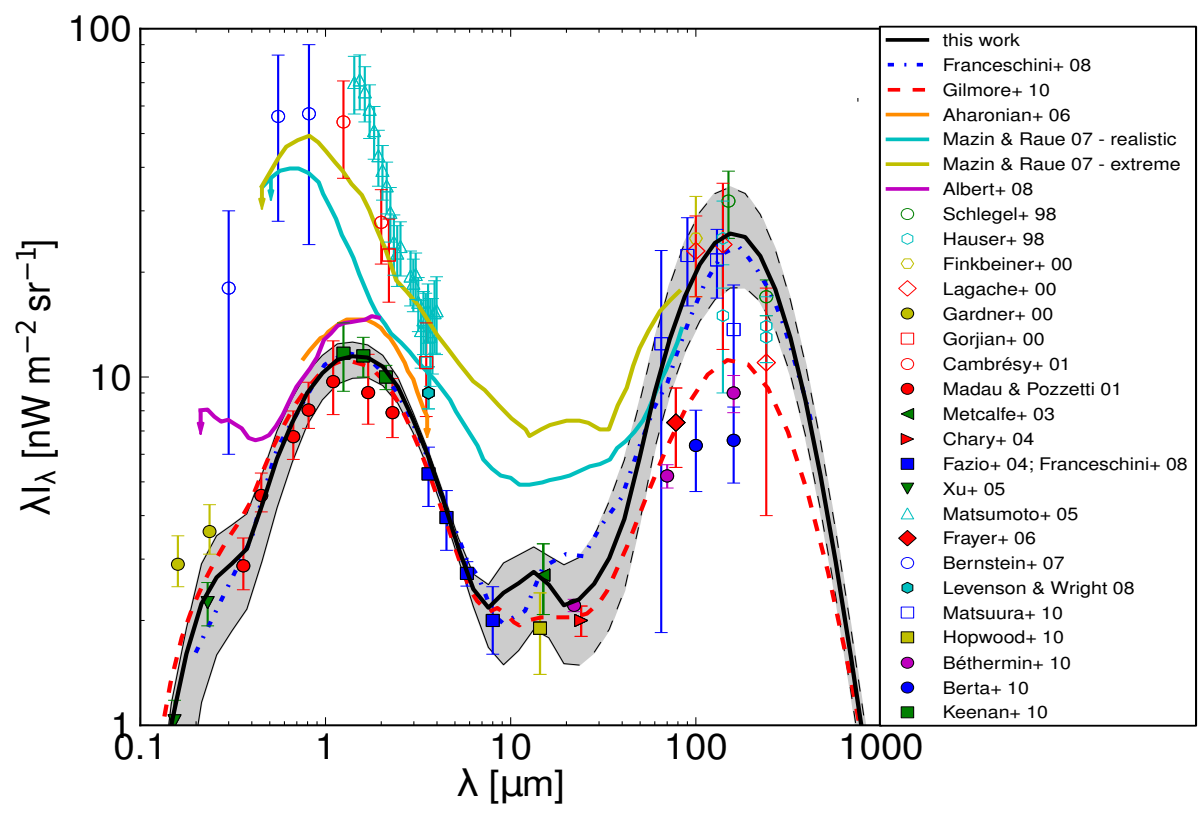

Figure 1: Spectral Energy Distribution (SED) of EBL represented through direct data, data from galaxy counts, upper limits from gamma-ray astronomy and recent EBL modelings. The solid-black line is the EBL calculated by the fiducial extrapolation of the galaxy-SED-type fractions for $\mathrm{z}>1$ by [8] while the shadow area indicates its uncertainties. The colored solid lines are upper limits from gamma-ray astronomy using different blazars. The dotted/dashed blue line and the dashed red line are the predictions from the models by [9] and [10], respectively; from [8].

In general, if the intrinsic spectrum was sufficiently well known, the EBL abundance could be effectively measured by comparing intrinsic (namely as emitted originally by the source) with observed spectra (namely as observed at Earth). Historically, diverse approaches have been pursued in this direction, but all relied on important, non-trivial assumptions of the intrinsic properties of the source, such as the capability to pinpoint the physical mechanism producing gamma rays. In particular, the techniques that I will discuss in the following are based on the absence of pile-up features in the Very-High-Energy (VHE; E $100 \mathrm{GeV}$ ) spectra (section 2.1), the overall description of the blazar SED in the framework of the synchrotron self-Compton (SSC) and external Compton (EC) modeling (section 2.2), and the extrapolation of the Fermi-LAT spectrum to the higher energies of the VHE regime (section 2.3). Blazars, however, are characterized by a broad range of possible spectra and our still incomplete understanding of their radiation mechanisms constitutes a main issue for the assumptions made to predict their intrinsic gamma-ray spectra. Besides, alternative scenarios propose further complications involving new fundamental physics or the interaction of the blazar radiation with the intergalactic medium. The former specifically refers to axion-like particle speculation [see e.g. 11] while the latter hypothesizes that the observed gamma rays are due to secondary emission resulting from the development of electromagnetic cascades originated by blazar cosmic rays in the intergalactic medium [12]. Both effects could result in an increase the effective mean free path of $\mathrm{TeV}$ gamma rays, but so far no certain clue has been found favoring them. 


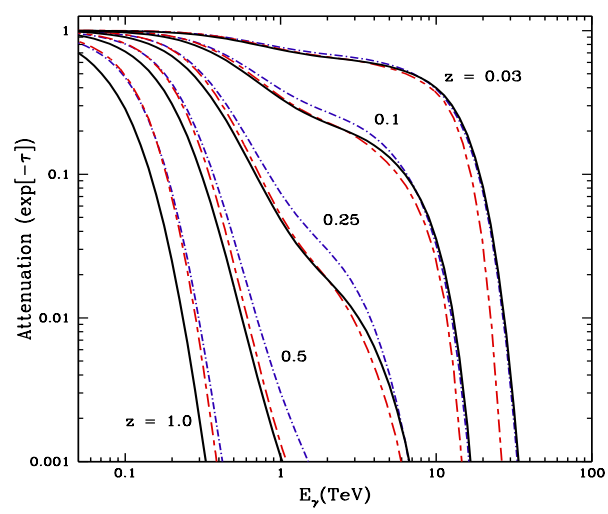

Figure 2: The attenuation $\mathrm{e}^{-\tau}$ of gamma rays vs. gamma-ray energy, for sources at different redshift. Increasing distance causes absorption features to increase in magnitude and appear at lower energies. The plateau seen between 1 and $10 \mathrm{TeV}$ at low redshift is a product of the mid-IR valley in the EBL spectrum. From [10].

\subsection{Hardness of the VHE spectra}

The blazar SED is characterized by two broad bumps located at lower and higher energies. The most common explanation is that their radiation arises from synchrotron and inverse Compton (IC) emission (for the lower and higher frequency bumps, respectively) from a population of relativistic electrons in the bulk motion of the relativistic jet. The seed photons for the IC process can be the synchrotron photons produced by the same electrons (synchrotron self-Compton models) or produced externally to the jet.

Following this framework, an upper limit on the EBL can be derived by requiring that the reconstructed gamma-ray photon number spectrum cannot be harder than $\Gamma=1.5$ in a powerlaw representation. This technique assumes that the blazar SED, as represented by the usual two bumps description, does not present up-turns or pile-up features at the higher energies (above the $\mathrm{GeV}$ range). Actually, using other suppositions within our current knowledge of blazars one can reasonable assume that the intrinsic spectrum at the highest frequencies is not harder than $\Gamma=$ 1.5. This limit is derived by shock acceleration models, where the hardest index obtained for the accelerated particles is 1.5. Similarly, in the case of protons interacting with ambient plasma, the resulting gamma-ray spectrum is supposed to have the same slope. Thus, the $\Gamma=1.5$ represents the reference value between actual observation and other theoretical speculation.

An outstanding result was obtained by the H.E.S.S. collaboration with a sample of unexpectedly hard-spectra blazars [13]. They extrapolated these spectra from low to high energies and found an energy-dependent attenuation feature in the high-z blazars 1ES1101-232 ( $\mathrm{z}=0.186$, see Figure 3) and H2356-309 ( $\mathrm{z}=0.165)$. The upper limit that was derived on the EBL at optical/near-infrared wavelengths pointed toward the lower estimates, close to the limits given by the integrated light of resolved galaxies. While suggesting a predominant contribution of direct starlight from galaxies to EBL, with more than $2 / 3$ of the EBL in the optical/near-infrared bands ascribable to single sources, it also excluded a large contribution from other sources, in particular from the Population III stars. In addition, this result indicated that intergalactic space was more transparent to gamma rays than 
what was previously thought.

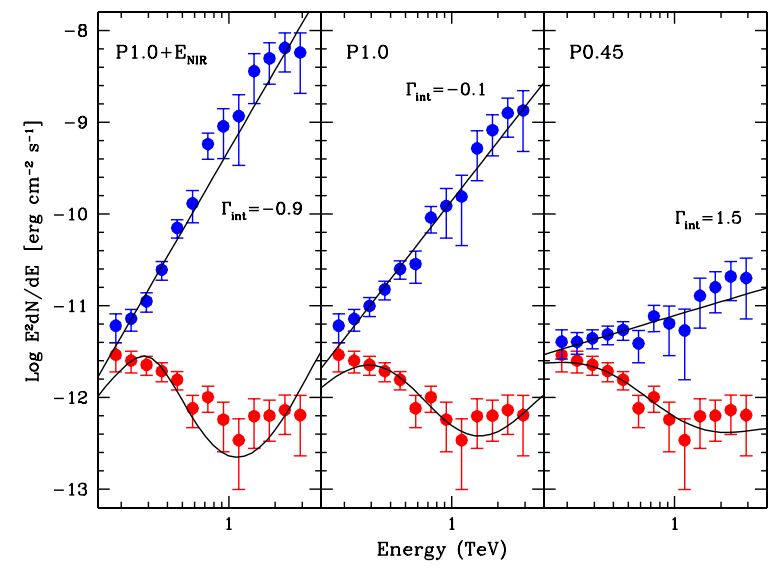

Figure 3: The H.E.S.S. spectra of 1ES1101-232, corrected for absorption with three different EBL SEDs. Red points indicate observed data, blue the absorption-corrected data; from [13].

\subsection{SED modeling of the blazar spectra}

So far the blazar SED has usually been adequately described in the framework of the synchrotron self-Compton and external Compton scenario. Thus, the radiation emitted in the MeV$\mathrm{GeV}$ range, which is observed by the Fermi-LAT, and the one emitted in the TeV range, observed by the Cherenkov telescopes, are supposed to be produced by the same particle populations [14]. In principle, if both emissions belong to the same inverse-Compton hump, the flux and spectrum at very high energies should always be lower and steeper than at HE. As a consequence, the extrapolation of the Fermi-LAT spectrum into the VHE band was considered either a good estimate or an upper limit for the intrinsic VHE spectrum.

One can therefore extrapolate the Fermi spectrum of local Universe sources $(z<0.2)$, which is not affected by EBL absorption, into the VHE band and compare it to current Cherenkov telescope observations. The correction of the VHE data needed to match the predicted intrinsic emission translates to an EBL upper limit. However recent detailed observations of blazar SEDs revealed that this assumption may be not be appropriate, at least in some cases. For instance, the case of Mkn 501 discussed in [15] pointed out a VHE spectrum with $\Gamma \sim 1.5-1.7$ (see Fig. 4). This could hint at the presence of a hard-spectrum VHE component capable of exceeding a softer HE spectrum, invalidating the aforementioned relation between the HE and VHE indexes (see previous Section 2.1).

Alternatively, one can use the full multiwavelength data and apply the SSC or EC modeling to predict the intrinsic VHE emission. This approach is more realistic than the previous one but, on the other hand, is strongly model-dependent. The availability of continuously improved data samples leads to more detailed descriptions of blazar SEDs. These are showing that in several cases classical models (e.g. single-zone SSC) are not completely adequate and often fail to reproduce the overall blazar properties. The picture is complicated further when considering the presence of a superposition of multiple spectral components in their synchrotron emission, which could be acting 


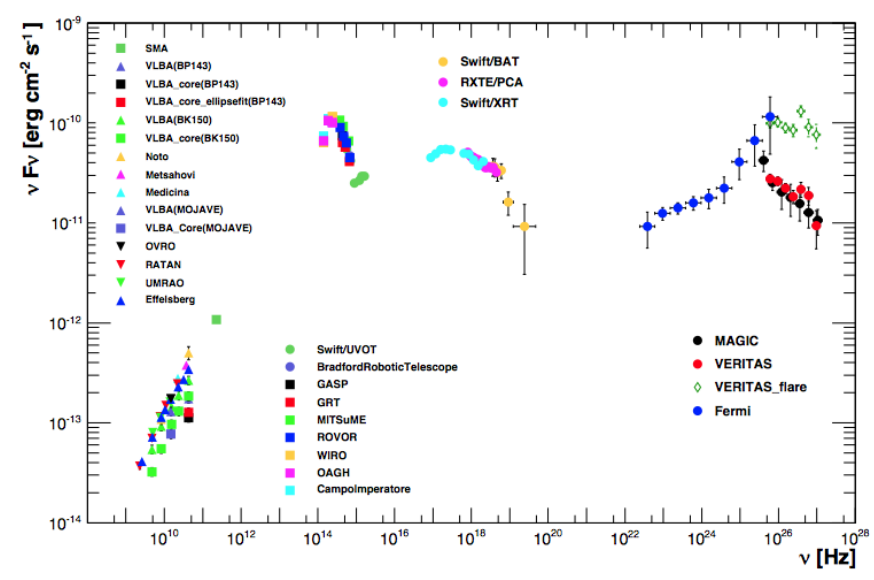

Figure 4: SED for Mkn 501 averaged over observations taken during the multifrequency campaign performed between 2009 March 15 (MJD 54905) and 2009 August 1 (MJD 55044). Interestingly, the FermiLAT spectrum (blue points) hints at a hardening toward the higher energies. Instruments and corresponding measured fluxes are reported in the legend; from [15].

especially during high-activity states and could be transposed by inverse Compton interaction into the gamma-ray band. Contextually, it is worthwhile to note that these approaches assume that the blazar gamma-ray spectrum should always be convex ${ }^{1}$. The inverse Compton peak extends over five decades in energy and it looks reasonable to expect that it could arise form a combination of different emission components. The superposition of their emission could led to a complex behavior and maybe result in an overall concave spectrum.

\subsection{Collective imprint in the Fermi-LAT spectra}

The Fermi-LAT band $(0.1-100 \mathrm{GeV})$ is essentially insensitive to EBL attenuation for the local Universe, whereas it can be used to test this effect at the less explored higher redshifts, given that the critical energy ${ }^{2}$ above which the effect starts to be significant can be written as $\mathrm{E}_{\text {crit }}(z) \sim$ $170(1+z)^{-2.38} \mathrm{GeV}$. Until recent times, searching for this deviation in single source spectra has been the most straightforward application, but it provided only loose constraints.

An innovative and powerful approach looked for the collective imprint of the EBL absorption in the combined gamma-ray spectra of a sample of blazars, as a cut-off that changes amplitude and energy with redshift. The study, based on a subset of 150 bright blazars at known redshift in the $1-500 \mathrm{GeV}$ band discovered an average spectral cut-off compatible with the EBL absorption and led to limits on Population III star formation (see Fig. 6). These are thought to be the first stars to appear in the Universe and responsible for the initial ionization of the intergalactic medium after the dark ages, as well as thought to have created the first metals and disperse them in the intergalactic medium. [16] constrained the redshift of maximum formation of low-metallicity stars to be at $z \geq 10$. The derived upper limit on its peak co-moving star-formation rate was of $0.5 \mathrm{M}_{\odot}$ $\mathrm{Mpc}^{-3} \mathrm{yr}^{-1}$, consistent with the peak star-formation rate of $0.2-0.6 \mathrm{M}_{\odot} \mathrm{Mpc}^{-3} \mathrm{yr}^{-1}$.

\footnotetext{
${ }^{1}$ We define a spectrum convex when the photon index $\Gamma$ decreases with energy.

${ }^{2} \mathrm{E}_{\text {crit }}$ is the critical energy above which more than $5 \%$ of the source photons are absorbed.
} 


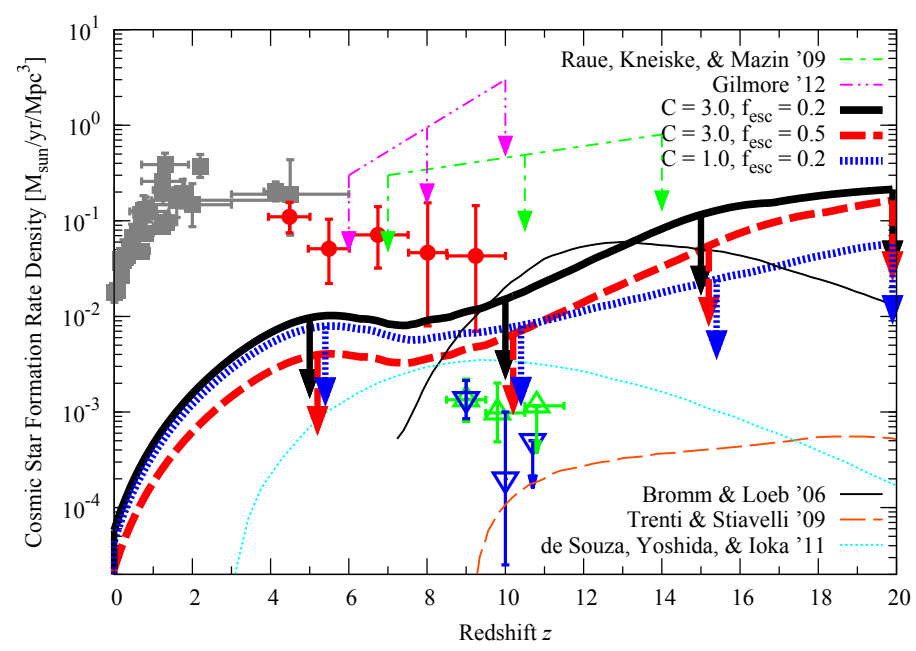

Figure 5: Limits on the Population III star formation history obtained combining constraints on the gammaray opacity, the optical depth for Thomson scattering and the neutral fraction of hydrogen; from [18].

The same idea has been subsequently applied to a sample of $\mathrm{TeV}$ blazars with data collected in the VHE band [17]. One of the major criticisms of this approach is that in principle the attenuation feature could be related to blazar intrinsic emission. However this would require that the blazar intrinsic properties evolves in an ad hoc manner, to mimic precisely the action of EBL attenuation with redshifts. Current gamma-ray data do not provide strong constraints on the Population III star formation history because the most distant object is still at $\mathrm{z}=4.35$ where the reionization process is completed [18]. Hopefully the new generation of gamma-ray instruments, namely the Cherenkov Telescope Array [CTA, 19], will observe sources beyond redshift 6 enabling exploration of the reionization epoch via the gamma-ray technique [20].

\section{Conclusions}

There is an overall good agreement between modern constraints of EBL intensity from ultraviolet to far-infrared wavelengths. These point in the direction of a low-level EBL, close to estimates derived from resolved galaxy counts and [9, 21, 22, 8, 10, 23], implying the highest transparency of the Universe to gamma rays allowed by standard physics [24]. EBL measurements inferred from gamma-ray studies have proven to be a valuable complement to direct techniques and have already yielded crucial insights on galaxy evolution, star formation activity, dust extinction processes and understanding cosmic structure formation and evolution [see e.g. Figure 5, 18].

Besides the important outcomes on stellar archaeology, a better characterization of EBL properties can be used also to probe other crucial astrophysical unknowns, such as intergalactic magnetic field strength and ultra-high-energy accelerators, as well as serve to explore more exotic physics scenarios, namely axion-like particle oscillations. A fruitful future is expected for the new generation of imaging atmospheric Cherenkov telescopes, namely CTA, with the promise of substantially increasing the actual population of $\mathrm{TeV}$ sources. This will hopefully offer a large sample of objects, also at higher redshift with respect to the current sample, to test EBL predictions and make progress also in related fields. 


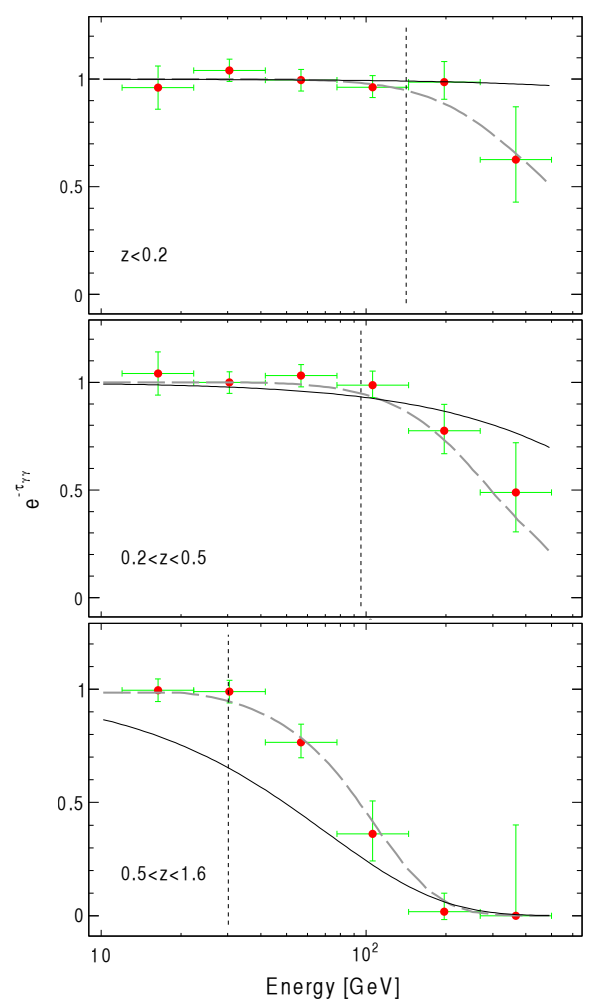

Figure 6: Absorption feature present in the spectra of BL Lac objects as a function of increasing redshift (data points, from top to bottom). The dashed curves show the attenuation expected for the sample of sources. The vertical line shows the critical energy below which less than 5\% of the source photons are absorbed by the EBL. The thin solid curve represents the best-fit model assuming that all the sources have an intrinsic exponential cut-off and that blazars follow the blazar sequence model of [25]; from [16].

\section{Acknowledgments}

The Fermi-LAT Collaboration acknowledges support from a number of agencies and institutes for both development and the operation of the LAT as well as scientific data analysis. These include NASA and DOE in the United States, CEA/Irfu and IN2P3/CNRS in France, ASI and INFN in Italy, MEXT, KEK, and JAXA in Japan, and the K. A. Wallenberg Foundation, the Swedish Research Council and the National Space Board in Sweden. Additional support from INAF in Italy and CNES in France for science analysis during the operations phase is also gratefully acknowledged. SB thanks D. J. Thompson for comments that improved the manuscript.

\section{References}

[1] M. G. Hauser and E. Dwek, The Cosmic Infrared Background: Measurements and Implications, Annu. Rev. Astron. Astr. 39 (2001) 249-307, [astro-ph/ 0105539 ].

[2] J. R. Primack, J. S. Bullock, and R. S. Somerville, Observational Gamma-ray Cosmology, in High Energy Gamma-Ray Astronomy (F. A. Aharonian, H. J. Völk, and D. Horns, eds.), vol. 745 of American Institute of Physics Conference Series, pp. 23-33, Feb., 2005.

astro-ph/0502177. 
[3] V. Bromm and R. B. Larson, The First Stars, Annu. Rev. Astron. Astr. 42 (Sept., 2004) 79-118, [astro-ph/0311019].

[4] R. J. Gould and G. P. Schréder, Opacity of the Universe to High-Energy Photons, Physical Review 155 (Mar., 1967) 1408-1411.

[5] F. W. Stecker, O. C. de Jager, and M. H. Salamon, TeV gamma rays from 3C 279 - A possible probe of origin and intergalactic infrared radiation fields, Astrophys. J. Lett. 390 (May, 1992) L49-L52.

[6] L. Costamante, Gamma-Rays from Blazars and the Extragalactic Background Light, International Journal of Modern Physics D 22 (Nov., 2013) 30025, [arXiv:1309. 0612].

[7] A. Domínguez, An empirical approach to the extragalactic background light from AEGIS galaxy SED-type fractions, in IAU Symposium (R. J. Tuffs and C. C. Popescu, eds.), vol. 284 of IAU Symposium, pp. 442-445, Aug., 2012. arXiv: 1111. 4435.

[8] A. Domínguez, J. R. Primack, D. J. Rosario, F. Prada, R. C. Gilmore, S. M. Faber, D. C. Koo, R. S. Somerville, M. A. Pérez-Torres, P. Pérez-González, J.-S. Huang, M. Davis, P. Guhathakurta, P. Barmby, C. J. Conselice, M. Lozano, J. A. Newman, and M. C. Cooper, Extragalactic background light inferred from AEGIS galaxy-SED-type fractions, Mon. Not. R. Astron. Soc. 410 (Feb., 2011) 2556-2578, [arXiv: 1007.1459 ].

[9] A. Franceschini, G. Rodighiero, and M. Vaccari, Extragalactic optical-infrared background radiation, its time evolution and the cosmic photon-photon opacity, Astron. Astrophys. $\mathbf{4 8 7}$ (Sept., 2008) 837-852, [arXiv:0805.1841].

[10] R. C. Gilmore, R. S. Somerville, J. R. Primack, and A. Domínguez, Semi-analytic modelling of the extragalactic background light and consequences for extragalactic gamma-ray spectra, Mon. Not. R. Astron. Soc. 422 (June, 2012) 3189-3207, [arXiv: 1104.0671 ].

[11] Tavecchio et al., Photons into axion-like particles conversion in Active Galactic Nuclei, arXiv:1406.2303 (June, 2014) [arXiv:1406.2303].

[12] Essey et al., Secondary Photons and Neutrinos from Cosmic Rays Produced by Distant Blazars, Phys. Rev. Lett. 104 (Apr., 2010) 141102, [arXiv:0912.3976].

[13] F. Aharonian et al., A low level of extragalactic background light as revealed by $\gamma$-rays from blazars, Nature 440 (Apr., 2006) 1018-1021, [astro-ph/ 0508073 ].

[14] P. S. Coppi and F. A. Aharonian, Understanding the spectra of TeV blazars: implications for the cosmic infrared background, Astroparticle Physics 11 (June, 1999) 35-39, [astro-ph/9903160].

[15] A. A. Abdo et al., Insights into the High-energy $\gamma$-ray Emission of Markarian 501 from Extensive Multifrequency Observations in the Fermi Era, Astrophys. J. 727 (Feb., 2011) 129, [arXiv:1011.5260]. 
[16] M. Ackermann et al., The Imprint of the Extragalactic Background Light in the Gamma-Ray Spectra of Blazars, Science 338 (Nov., 2012) 1190-, [arXiv:1211.1671].

[17] H.E.S.S. Collaboration, Measurement of the extragalactic background light imprint on the spectra of the brightest blazars observed with H.E.S.S., Astron. Astrophys. 550 (Feb., 2013) A4, [arXiv:1212.3409].

[18] Y. Inoue, Y. T. Tanaka, G. M. Madejski, and A. Domínguez, Upper Bound on the First Star Formation History, Astrophys. J. Lett. 781 (Feb., 2014) L35, [arXiv: 1312.6462].

[19] M. Actis, G. Agnetta, F. Aharonian, A. Akhperjanian, J. Aleksić, E. Aliu, D. Allan, I. Allekotte, F. Antico, L. A. Antonelli, and et al., Design concepts for the Cherenkov Telescope Array CTA: an advanced facility for ground-based high-energy gamma-ray astronomy, Experimental Astronomy 32 (Dec., 2011) 193-316, [arXiv: 1008.3703 ].

[20] Y. Inoue, S. Inoue, M. A. R. Kobayashi, R. Makiya, Y. Niino, and T. Totani, Extragalactic Background Light from Hierarchical Galaxy Formation: Gamma-Ray Attenuation up to the Epoch of Cosmic Reionization and the First Stars, Astrophys. J. 768 (May, 2013) 197, [arXiv:1212.1683].

[21] J. D. Finke, S. Razzaque, and C. D. Dermer, Modeling the Extragalactic Background Light from Stars and Dust, Astrophys. J. 712 (Mar., 2010) 238-249, [arXiv: 0905.1115 ].

[22] T. M. Kneiske and H. Dole, A lower-limit flux for the extragalactic background light, Astron. Astrophys. 515 (June, 2010) A19, [arXiv:1001.2132].

[23] F. W. Stecker, M. A. Malkan, and S. T. Scully, A Determination of the Intergalactic Redshift-dependent Ultraviolet-Optical-NIR Photon Density Using Deep Galaxy Survey Data and the Gamma-Ray Opacity of the Universe, Astrophys. J. 761 (Dec., 2012) 128, [arXiv:1205.5168].

[24] A. Domínguez, M. A. Sánchez-Conde, and F. Prada, Axion-like particle imprint in cosmological very-high-energy sources, J. Cosmol. Astropart. P. 11 (Nov., 2011) 20, [arXiv:1106.1860].

[25] G. Fossati, L. Maraschi, A. Celotti, A. Comastri, and G. Ghisellini, A unifying view of the spectral energy distributions of blazars, Mon. Not. R. Astron. Soc. 299 (Sept., 1998) 433-448, [astro-ph/9804103]. 